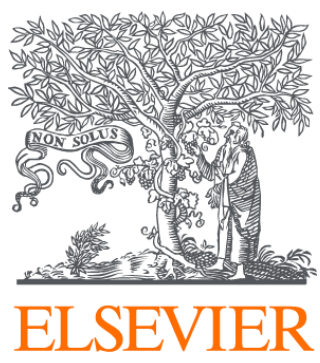

Since January 2020 Elsevier has created a COVID-19 resource centre with free information in English and Mandarin on the novel coronavirus COVID-

19. The COVID-19 resource centre is hosted on Elsevier Connect, the company's public news and information website.

Elsevier hereby grants permission to make all its COVID-19-related research that is available on the COVID-19 resource centre - including this research content - immediately available in PubMed Central and other publicly funded repositories, such as the WHO COVID database with rights for unrestricted research re-use and analyses in any form or by any means with acknowledgement of the original source. These permissions are granted for free by Elsevier for as long as the COVID-19 resource centre remains active. 


\title{
An experimental study of $\mathrm{RyO}$ Kit for Covid-19 information sharing in rural islands of Indonesia
}

\author{
Irfan Syamsuddin ${ }^{\mathrm{a}, *}$, Irmawati $^{\mathrm{a}}$, Syafaruddin ${ }^{\mathrm{b}}$ \\ a Department of Computer and Network Engineering, State Polytechnic of Ujung Pandang, Makassar, Indonesia \\ ${ }^{\mathrm{b}}$ Department of Electrical Engineering, Hasanuddin University, Makassar, Indonesia \\ Received 19 July 2020; received in revised form 26 January 2021; accepted 3 February 2021 \\ Available online 11 February 2021
}

\begin{abstract}
Indonesia is among the most suffering countries due to Covid-19 pandemic. The government is still challenged to manage the delivery of Covid-19 related information since many of inhabitants living in rural islands. This aims to offer low cost and simple solution for government in delivering Covid-19 information in rural islands without adequate telecommunications infrastructure. The technical solution is based on Raspberry Pi technology called RyO (Raspberry Outernet) Kit. We examined RyO Kit based on four parameters namely signal strength, signal quality, bit rate and quota. Final results confirmed RyO Kit is appropriate solution to deal with the issue.

(C) 2021 The Korean Institute of Communications and Information Sciences (KICS). Publishing services by Elsevier B.V. This is an open access article under the CC BY-NC-ND license (http://creativecommons.org/licenses/by-nc-nd/4.0/).
\end{abstract}

Keywords: RyO Kit; Raspberry Pi; Rural island; Information sharing; Covid-19

\section{Introduction}

Indonesia is known as the largest archipelago country in the world. With more than 16,000 islands, it is very difficult for Indonesian government to satisfy the communication needs of the people equally particularly for those who live on rural islands. [1]. Like other developing countries, Indonesia has limited budget to satisfy all rural areas that are scattered by the sea to enjoy excellent quality of communications in affordable price. People who live particularly in rural islands could not enjoy appropriate communications as their counterparts in urban areas of Indonesia. Gap of digital divide in Indonesia is significantly wider than other developing countries which in turn it affects many aspects of people live such as inequality of economic access, poor of education facilities and particularly lack of health information such as Covid-19, due to unavailability of adequate telecommunication infrastructure in many islands [2].

Since the outbreak of the Covid-19 pandemic in January 2020 until today where more than 56,000 people are infected worldwide, Indonesia is one of the countries with a very rapid

\footnotetext{
* Corresponding author.

E-mail address: irfans@poliupg.ac.id (I. Syamsuddin).

Peer review under responsibility of The Korean Institute of Communications and Information Sciences (KICS).
}

spread of Covid-19 [3]. The coronavirus pandemic in Indonesia begins with the discovery of patients with coronavirus 2019 (Covid-19) on March 2, 2020 [4] (see Fig. 1).

As of 4 April 2020, 2092 positive cases of Covid-19 have been confirmed, with 181 of them dead and 150 recovered, while about 11 new cases reported in daily basis [4]. As shown in Fig. 2, cases of Covid-19 has been severely affected 32 provinces out of a total of 34 provinces in Indonesia (red areas) and only 2 others without any reported cases (blue areas) as seen in Fig. 2 [4].

Apart from delay response by government to consider Covid-19 as a serious nation-wide issue [5], inhabitants in remote islands without communication supports are in serious danger. Such lack conditions where no available communication infrastructure have pushed doctors, nurses and health staffs to travel by their own cost (no funding support from government yet) to the rural island and talk with local people there physically although without using hazmat uniform [5]. If appropriate communication facilities exist, they (health officials) would be able to share related information with people rural islands to reduce direct contact that possibly affect them.

The motivation for this research derived from our concern regarding the difficulties faced by the government, in this case the health officials, in conveying health related information directly by visiting remote islands especially during current 


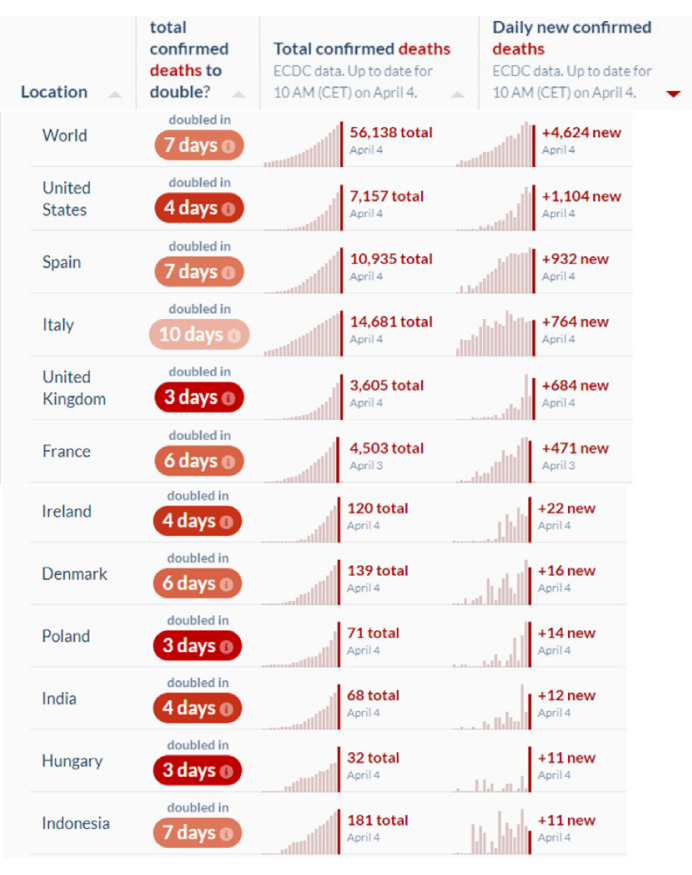

Fig. 1. World statistics of Covid 19.

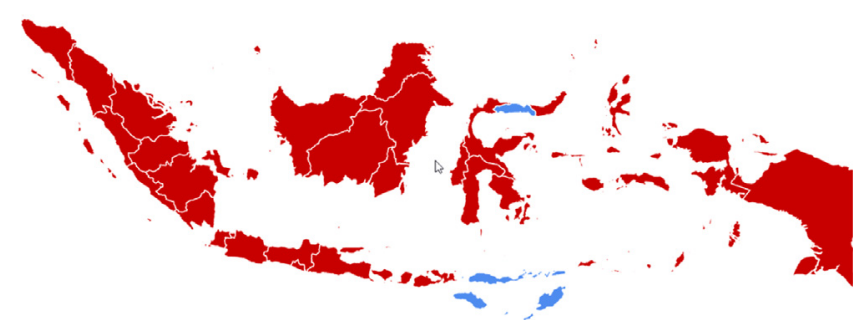

Fig. 2. Spread of Covid-19 in Indonesian archipelago.

Covid-19 pandemic. Through this research we aim to contribute in reducing the communication gap in remote island areas by combining Outernet technology and Raspberry Pi adapted to specific conditions at the location. It is believed that this will help in reducing physical contact which is very Covid-19 risky both for health officials as well as for people in rural island as currently occurs. To the best of authors' knowledge there has been no similar approach to deal with the issue of delivering Covid-19 related information in rural areas in Indonesia as presented in this paper.

The paper is organized as follows. The second section describes related studies followed by the methodology applied from initial system setup to evaluation phases in section three. It is then followed by section four of analysis and discussion. Finally, the conclusion section summarizes our study.

\section{Related studies}

Death toll due to Covid-19 surged in a relatively short time around the world. Covid-19 affects all countries, regardless of whether the country is a developed or developing country.

In developed countries, advanced technology-based efforts have been deployed to overcome a number of problems caused by Covid-19. In recent study, Fong, et al. (2021) argued various ways in which artificial intelligence or AI has extensively been used to increase the validity and speed of health decision support systems in dealing with Covid-19 outbreak [6].

Besides, Jad and Singh [7] described the application of artificial intelligence (AI) technology to enhance the quality of robot surveillance for Covid-19 outbreak. This is also in line with research by with Hussain, et al. [8], who emphasize the power of AI during Covid-19 pandemic to formulate effective of public health interventions and simplify real-time epidemiological Covid-19 data collection.

In China, as described in [9] AI has also been combined with Deep Learning to enhance diagnosis and trend prediction of Covid-19. Another combination between AI and Big Data is studied by Bragazzi, et al. [10] to deal with huge amount of data relating to Covid-19 surveillance and show enhanced results in real-time Covid-19 outbreaks monitoring and forecasting.

When developed countries have implemented various advanced technologies in dealing with Covid-19 as mentioned above, unfortunately developing countries such as Indonesia still left behind mainly due to fundamental obstacles, such as unavailability of adequate communication facilities to support disseminating information about Covid-19 to those in remote islands.

Until now, radio broadcasts from government official channel (Radio Republic of Indonesia or RRI) is the only medium for the people there to obtain information, and in such severe Covid 19 pandemic is unreliable.

As reported by Abiad and Teipelke [11] since the end of the economic crisis in the 1990s, Indonesia has been experiencing an old problem, namely a significant imbalance of economic growth between urban and rural areas, which was marked by a lack of investment in telecommunications infrastructure. In the end, it creates information and social gaps between urban and rural areas, as currently happening during the Covid-19 pandemic.

Within these limitations, the government is still looking for alternative solutions besides building telecommunications towers on remote islands, so that they can enjoy internet access which is urgent in the dissemination of current Covid-19 information.

From the perspective of alternative internet provision, there are three technologies that have the potential to be examined for this case, they are Netsukuku [7,12], Loon [8,13] and Outernet $[9,14]$ projects.

Netsukuku aims to establish distributed network, anonymous and censorship-free, fully independent internet access without ISP [7,12]. Unfortunately it requires complex mechanisms which make it difficult to be applied in large scale networks. On the other hand, Loon which also known as Google Balloon was considered as a great solution for digital divide with strong support by Google $[8,13]$. However, it is not effective for the case of Indonesia islands. Outernet is another alternative that utilize satellite coverage as potential solution for filling communication gap in archipelago country with low cost hardware $[9,14]$. However it requires developing specific kit to enable the communications. 
Compared to Netsukuku and Loon, Outernet is preferable in our study due to four reasons. Firstly, Outernet utilizes the availability of satellite signal which covers most of areas in the world including rural islands. Secondly, Outernet provides free software and allows using any hardware to operate and therefore it is considered cost effective than Loon and Netsukuku. Thirdly, compare to Loon with very limited users in Africa and New Zealand or Netsukuku with small group of users in Europe, Outernet has several ongoing projects supported by many active user groups in Asia and North and Latin America [15]. Finally, Outernet kit might be deployed by using Raspberry-Pi which is available in local electronic market and affordable in terms of price [15].

Hence, we propose a combined Raspberry Pi and Outernet Kit called RyoKit to bridge the communications gap that currently hinder the effective spread of Covid-19 information to those who live in rural islands in Indonesia.

\section{Methodology}

To answer the research question, we construct the following research methodology based on [16]. It is formulated through six main steps as follows, hardware preparation, software installation, searching for parabolic coordinates, setup the system coordinates, testing four parameters, and finally apply the findings into RyoKit. for evaluation in black box testing and finally analysis of the findings (Fig. 3).

First of all, construct the system of RyO Kit as depicted in Fig. 4. Raspberry Pi is the main component of the systems since it is considered as the most viable technology to realize this study. In addition, Raspberry Pi has been widely used to establish many advanced electronic projects in various forms such as smart home, education, health and industrial applications from test bed [17] to real world implementations [18]. In this case, we use Raspberry Pi B+ type and select several components to construct the core of the systems based on Outernet standards as mentioned by Karim, et al. [14] and Makhduma, et al. [15]. The components are as follows:

- Parabola satellite dish (we used $60 \mathrm{~cm}$ ex-parabolic TV) with LNB or Low Noise Block for Ku band.

- DVB-Tuner with USB connection for satellite tuner

- HDMI cable for display connection

- USB keyboard

- Monitor with HDMI port

- Micro USB cable or AC power adaptor for Raspberry Pi

- SD card (16GB)

- Wireless router

- Laptop computer for client

- Android mobile for client

Secondly, after activating and running Raspberry Pi operating systems we perform installation of Outernet software. The software is called ORx which is available for free from Outernet official website.

Thirdly, after ORx running well in Raspberry Pi, we activate DVB tuner which is directly connected with the satellite dish to perform coordinate searching. In this step, the searching procedures are conducted several times until we obtain three different active coordinates. In order to ensure parabolic dish pointing to correct orbital position of AsiaSat 5, there are several trial and errors until the correct position found and then locked. Once all required components already determined, then we moved to the next step. We have formulated mechanism of parabolic pointing setup and obtained are three setting point as mentioned in [19]. These three setting points will be used for further evaluation step.

Then, in the next step, based on the coordinates obtained from previous step, we perform parameter evaluation. The evaluation is essential to ensure whether or not RyO Kit is working properly. According to the guidance from Outernet developer $[14,15,20]$ that there are four parameters should be examined, namely signal strength, signal quality, bit rate and quota, in order to ensure the successful of RyO Kit. In this step black box evaluation is conducted against the four parameters against three setting point already formulated in [19].

Finally, comparative analysis is performed to determine the best performance. Then the result is applied to $\mathrm{RyO}$ Kit to ensure the best performance of RyO Kit that is technically ready for real implementation for delivering Covid-19 information in rural islands of Indonesia.

\section{Analysis and discussion}

The crucial factor in this research is to determine the most accurate position of parabola dish. It is also challenging task since receiver device's sensitivity might be different one another according to which satellite covers the region (see Fig. 5).

To assist this process, several fundamental information are required which are obtained from www.satbeams.com, a dedicated website providing useful data collected from all currently orbiting satellites, in this case AsiaSat 5.

The information is very important to ensure the process of pointing is on the correct path. It includes Azimuth angle, Elevation and Inclination. Includes Tilt LNB or LNB angle position, suggested dish/parabolic size and exact pointing location coordinates and how far the satellite is from the satellite dish. Once the parabolic dish appointed to the right position, the ORx software indicates the existing link.

At this stage, we are ready to perform visibility study of RyO Kit. For this purpose, we have determined three setting points on different geographical locations labeled A, B, and C [19] from which we will further examine four parameters of Outernet whether or not satisfied by our RyO Kit. Once the first point (A) already settled, RyO Kit might start to download Outernet library contents as accessible via browser, later after finishing to collect all required data, the same procedures applied for B and C setting points.

It took less than five minutes then RyO Kit started showing connection indicators. This means, the kit has established connection with the AsiaSat 5 which also means connected to Outernet server. Then, RyO Kit showed a dashboard web working on port 100 over localhost, from which Outernet GUI might be accessed. 


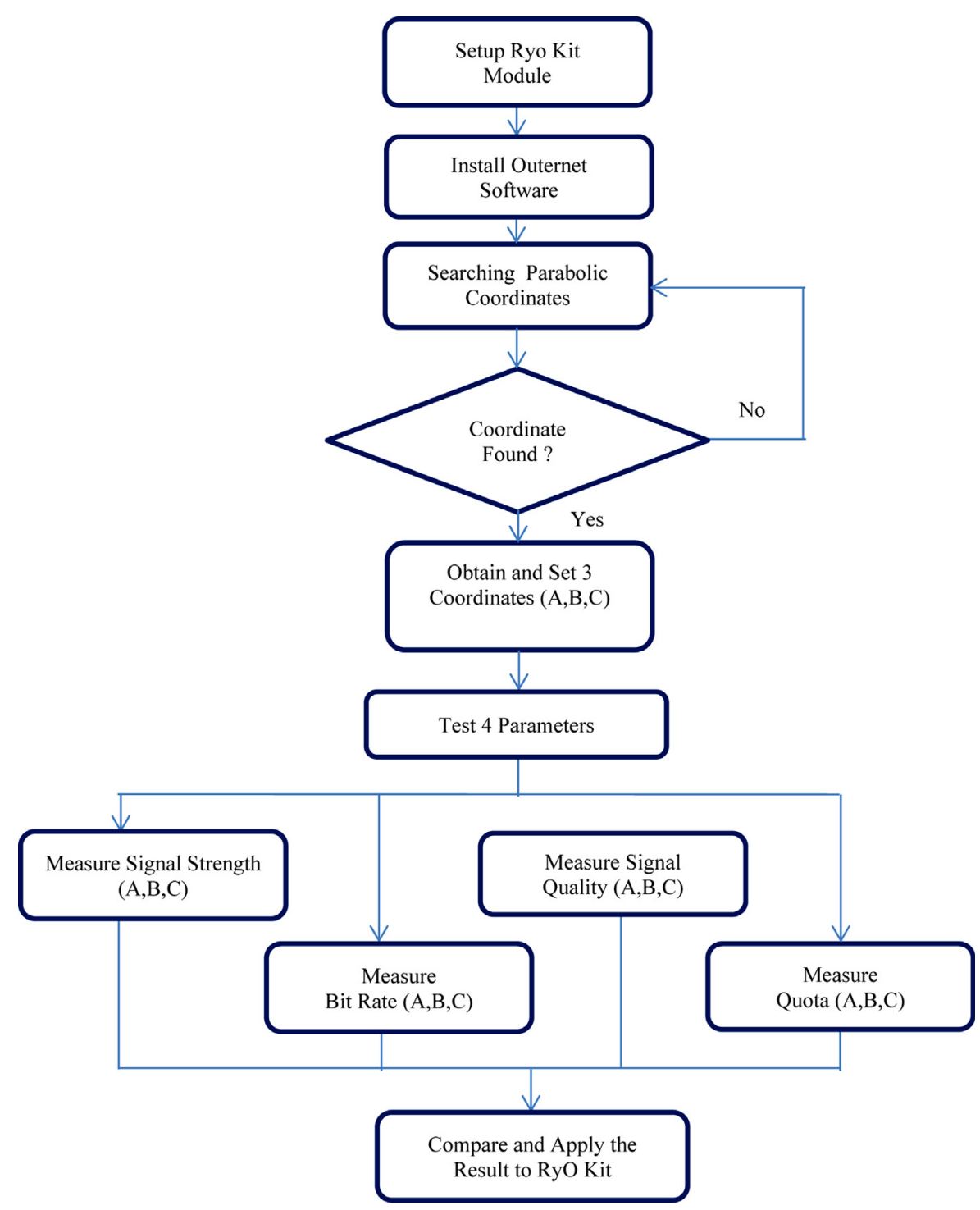

Fig. 3. Methodology applied in the study.

Outernet GUI providers several drop down links and one of them is tuner setting where all connection parameters are presented including an automatic downloaded content. The download process for the content might be viewed at the dashboard menu in the tuner settings menu (Fig. 6).

In tuner settings, it is clearly found that the GUI presents four parameters that are used to determine whether the RyO Kit works fine or not.

The following table and figures show our daily data within two weeks of experiment for all parameters (signal strength, signal quality, bit rate and quota).

First of all, we perform evaluation on signal strength parameter which defines how strong the signal from Outernet server acquired by RyO Kit. According to Karim, et al. [14] the minimum acceptable value for signal strength is $80 \%$ which is satisfied by all setting points in our case where minimum signal strength for A, B and C are $87 \%, 90 \%$ and $86 \%$ respectively. On the other hand, the maximum values are $91 \%$ for A, $94 \%$ for B and $93 \%$ for C (Table 1). As a result, it is

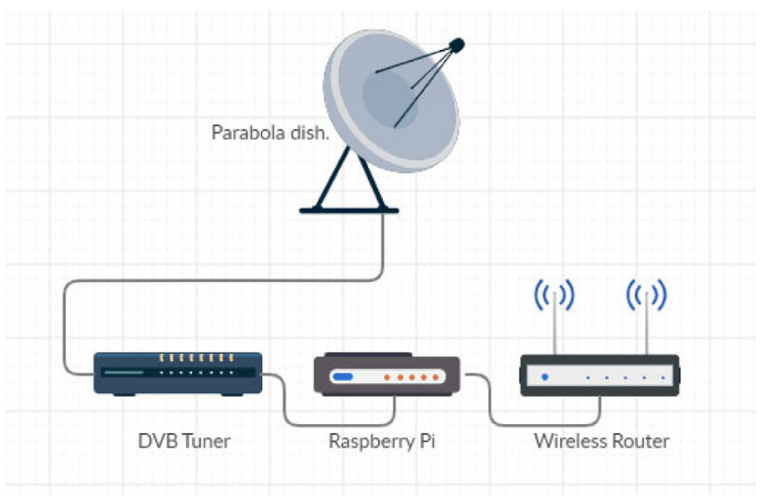

Fig. 4. System design of RyO Kit.

obviously found that setting point of B outperformed others by having highest average signal strength of $92.07 \%$ (Fig. 7).

The next parameter to be evaluated is signal quality which determines the goodness level of signal obtained by RyO Kit. 


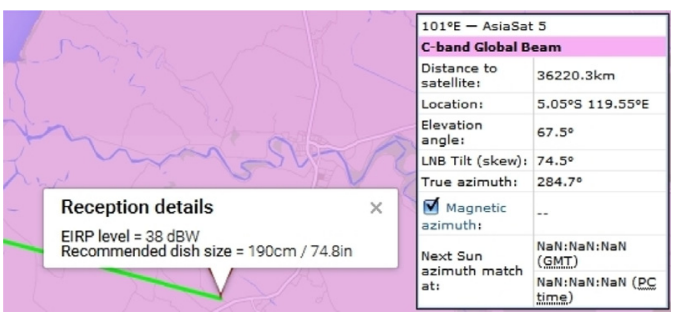

Fig. 5. Sunbeam information for AsiaSat 5.

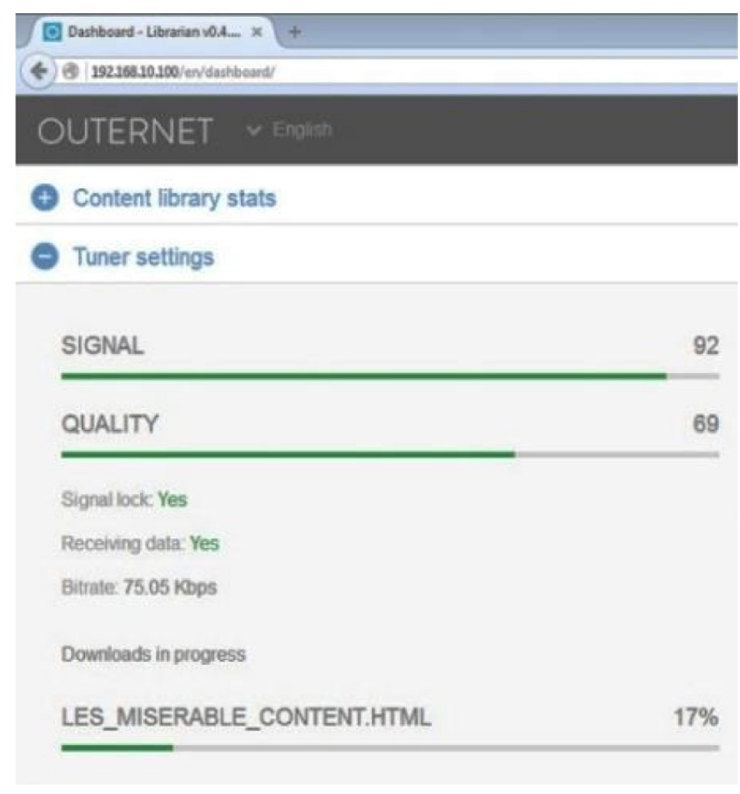

Fig. 6. Outernet connections established.

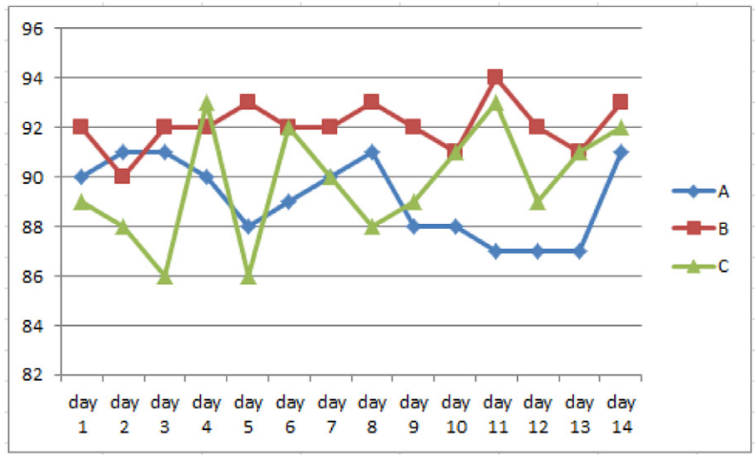

Fig. 7. Signal strength.

In similar way to previous parameter evaluation, we finally obtained daily data for all three setting points of $\mathrm{A}, \mathrm{B}$, and $\mathrm{C}$. In terms of minimum values, we got $65 \%, 67 \%$ and $63 \%$ for $\mathrm{A}, \mathrm{B}$, and $\mathrm{C}$ respectively. In this case, only $\mathrm{A}$ and $\mathrm{B}$ satisfy the standard of minimum signal quality of $65 \%$ according to $[14,15]$. In terms the maximum value, we found that $\mathrm{A}$ is $69 \%, \mathrm{~B}$ is $71 \%$ and $\mathrm{C}$ is $69 \%$ (Table 1). Finally, it is clearly seen that setting point of $\mathrm{B}$ shows better performance than $\mathrm{A}$ and $C$ with average value for signal strength of $69 \%$ (Fig. 8).

The third parameter is bit rate. Bit rate is a parameter to determine the speed of data transferred from Outernet server

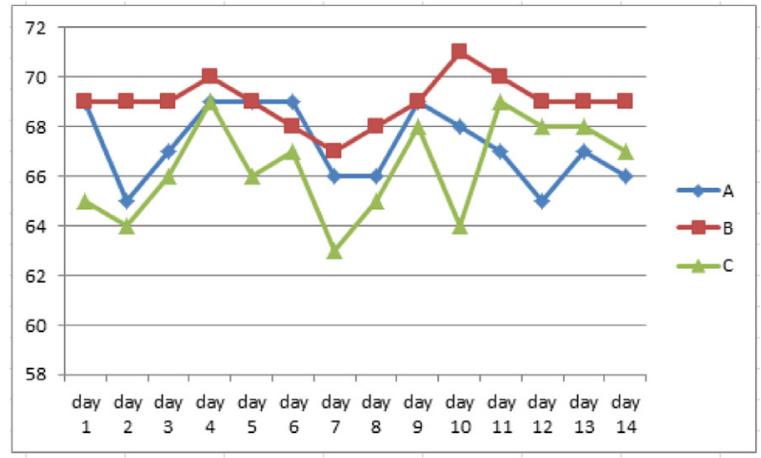

Fig. 8. Signal quality.

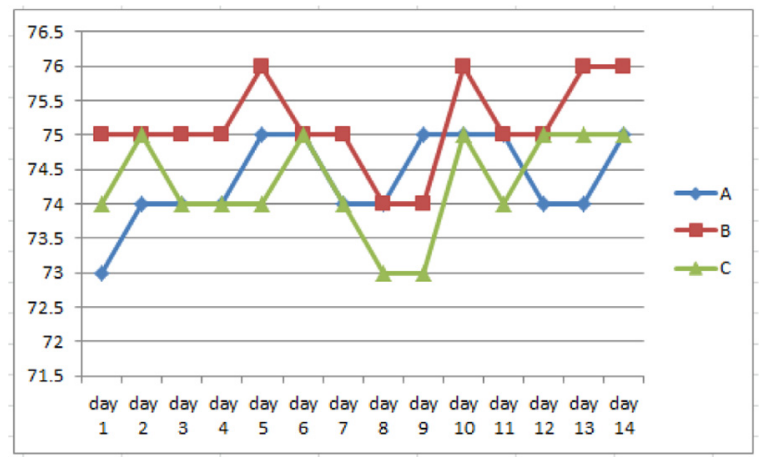

Fig. 9. Bit rate.

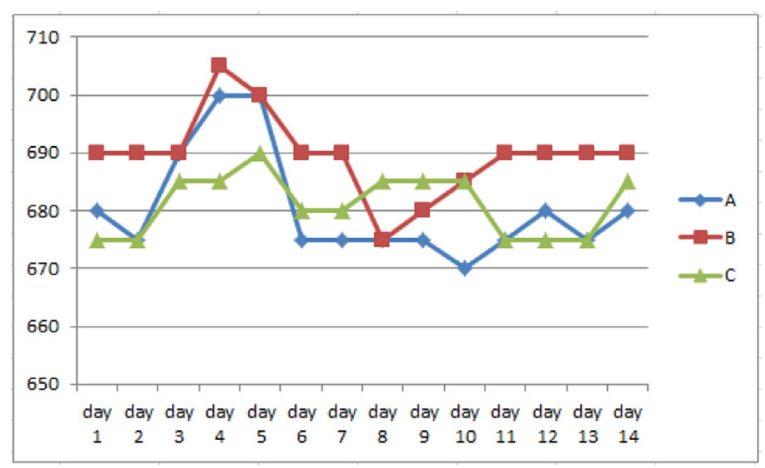

Fig. 10. Quota.

to RyO Kit. Basically, minimum of $70 \mathrm{Kbps}$ is acceptable for appropriate data transfer according to the developer of Outernet [14]. Based on our experiment in 14 days, it is found that all setting points show acceptable bit rate value of 73 Kbps, $74 \mathrm{Kbps}$ and $73 \mathrm{Kbps}$ for $\mathrm{A}, \mathrm{B}$, and $\mathrm{C}$ respectively. Besides, the maximum bit rate for setting points $\mathrm{A}, \mathrm{B}$, and $\mathrm{C}$ are $75 \mathrm{Kbps}, 76 \mathrm{Kbps}$ and $75 \mathrm{Kbps}$ in that order (Table 1). As can be seen in Fig. 9, the performance of setting point of $B$ is slightly better than $\mathrm{A}$ and $\mathrm{C}$ which accounted for $75.14 \mathrm{Kbps}$.

Finally, the last evaluated parameter is how many quotas could be obtained by RyO Kit. In this regards, all setting points show far beyond standard of Outernet quota (minimum 600 $\mathrm{MB}$ ) [9]. While the minimum quota obtained for A, B and C are $670 \mathrm{MB}, 675 \mathrm{MB}$ and $675 \mathrm{MB}$ respectively, the maximum ones are $700 \mathrm{MB}, 705 \mathrm{MB}$ and $690 \mathrm{MB}$ respectively (Table 1). 
Table 1

Evaluation results of four parameters from three locations.

\begin{tabular}{|c|c|c|c|c|c|c|c|c|c|c|c|c|}
\hline \multirow[t]{3}{*}{ Day } & \multicolumn{12}{|c|}{ Parameters } \\
\hline & \multicolumn{3}{|c|}{ Signal strength } & \multicolumn{3}{|c|}{ Signal quality } & \multicolumn{3}{|c|}{ Bit rate } & \multicolumn{3}{|l|}{ Quota } \\
\hline & $\mathrm{A}$ & B & $\mathrm{C}$ & A & B & $\mathrm{C}$ & A & B & $\mathrm{C}$ & A & B & $\mathrm{C}$ \\
\hline 1 & 90 & 92 & 89 & 69 & 69 & 65 & 73 & 75 & 74 & 680 & 690 & 675 \\
\hline 2 & 91 & 90 & 88 & 65 & 69 & 64 & 74 & 75 & 75 & 675 & 690 & 675 \\
\hline 3 & 91 & 92 & 86 & 67 & 69 & 66 & 74 & 75 & 74 & 690 & 690 & 685 \\
\hline 4 & 90 & 92 & 93 & 69 & 70 & 69 & 74 & 75 & 74 & 700 & 705 & 685 \\
\hline 5 & 88 & 93 & 86 & 69 & 69 & 66 & 75 & 76 & 74 & 700 & 700 & 690 \\
\hline 6 & 89 & 92 & 92 & 69 & 68 & 67 & 75 & 75 & 75 & 675 & 690 & 680 \\
\hline 7 & 90 & 92 & 90 & 66 & 67 & 63 & 74 & 75 & 74 & 675 & 690 & 680 \\
\hline 8 & 91 & 93 & 88 & 66 & 68 & 65 & 74 & 74 & 73 & 675 & 675 & 685 \\
\hline 9 & 88 & 92 & 89 & 69 & 69 & 68 & 75 & 74 & 73 & 675 & 680 & 685 \\
\hline 10 & 88 & 91 & 91 & 68 & 71 & 64 & 75 & 76 & 75 & 670 & 685 & 685 \\
\hline 11 & 87 & 94 & 93 & 67 & 70 & 69 & 75 & 75 & 74 & 675 & 690 & 675 \\
\hline 12 & 87 & 92 & 89 & 65 & 69 & 68 & 74 & 75 & 75 & 680 & 690 & 675 \\
\hline 13 & 87 & 91 & 91 & 67 & 69 & 68 & 74 & 76 & 75 & 675 & 690 & 675 \\
\hline 14 & 91 & 93 & 92 & 66 & 69 & 67 & 75 & 76 & 75 & 680 & 690 & 685 \\
\hline Min & 87 & 90 & 86 & 65 & 67 & 63 & 73 & 74 & 73 & 670 & 675 & 675 \\
\hline $\operatorname{Max}$ & 91 & 94 & 93 & 69 & 71 & 69 & 75 & 76 & 75 & 700 & 705 & 690 \\
\hline Avg & 89.14 & 92.07 & 89.78 & 67.28 & 69 & 66.35 & 74.35 & 75.14 & 74.28 & 680.35 & 689.64 & 681.07 \\
\hline
\end{tabular}

Overall, setting point of B again shows the best performance among others in terms of quota by $689.64 \mathrm{MB}$ in average as depicted in Fig. 10.

Based on our examinations on four parameters, it is concluded that setting point of $\mathrm{B}$ is chosen for further RyO Kit implementation in the chosen location. The selected setting point shows excellent signal strength, signal quality, bit rate and quota for the purpose of sending Covid-19 related information to people in rural areas which in turn will help in controlling the spread of the pandemic in rural areas because RyO Kit solution could be replicated easily with less efforts and minimum cost in other rural islands both in Indonesia or other countries.

Particularly from health perspective, along with research findings by WHO about difficulties in disseminating health related information in archipelago countries [21], RyO Kit is a notable solution by Indonesia government in the case of delivering Covid-19 information in rural islands.

In comparison to radio broadcast which is currently in use in rural islands, RyO Kit has a number of advantages. First, RyO Kit is able to convey information in digital format while Radio is not. Second, communication via RyO Kit is not susceptible to weather changes, while radio generally degrades in quality in such conditions. Thirdly, RyO Kit allows two ways text, sound, video streaming communications while the current Radio is only one way sound communication.

Then, we compare our RyO Kit with conventional communication networks that require building telecom tower. From economics point of view, establishing RyO Kit is much more affordable for government than any other option such as establishing telecom towers. It is calculated that development of RyO Kit only costs less than USD 400 which is significantly cheaper than cost for building a telecom tower of minimum USD 20000 [22]. In addition, time required to develop RyO Kit is very short which approximately takes less than two weeks from setup until running the parameter testing. In

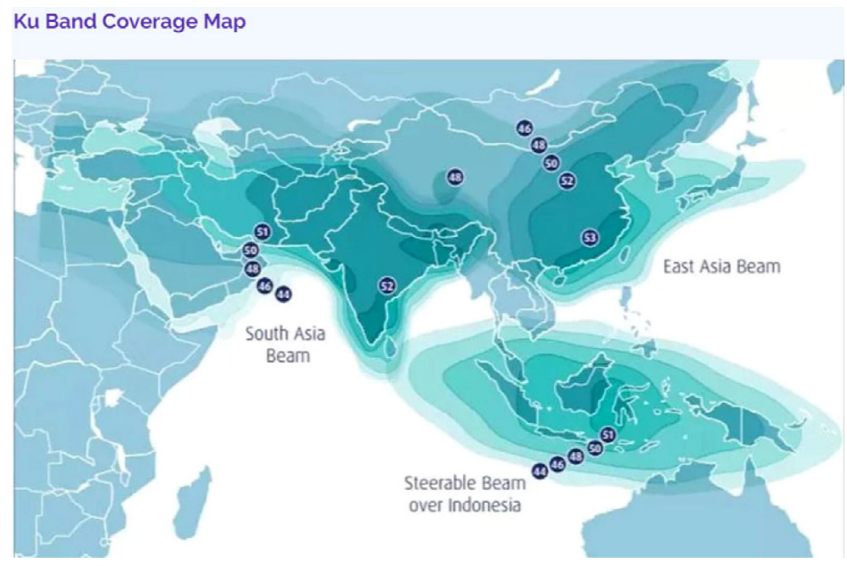

Fig. 11. AsiaSat 5 coverage. Source: https://www.ikomg.c om/asiasat5.

short, from economic and efficiency perspectives, RyO Kit has potential to be applied in broader scales to bridge digital divide in rural islands of Indonesia.

In addition, all regions of Republic of Indonesia are well covered by AsiaSat5 satellite, mostly with good coverage while few others with fair coverage as seen in Fig. 10. Therefore, $\mathrm{RyO}$ Kit has a great potential as a reliable communication media for rural islands for long period of time (see Fig. 11).

The following are two future recommendations in which RyO Kit would be used effectively by government to spread Covid-19 related information to people who live in rural islands.

Firstly, since radio broadcasting as the only communication facility available currently does not sufficient for delivering Covid-19 information in digital format, the implementation of RyO Kit in this case is a feasible option. Development cost of RyO Kit of less than USD 400 is affordable by government in 
cooperation with other parties such as CSR fund from companies, research cooperation with universities or crowd funding by local people. Moreover, successful RyO Kit applications in dissemination of Covid-19 information for surveillance and monitoring purposes in remote islands could be a reference for other developing countries that are experiencing the same problems during the Covid-19 pandemic.

Secondly, since all schools have been closing since four weeks ago (the time when the manuscript firstly written), then all students in Indonesia should study at home through the Internet. Unfortunately, students who live in rural islands could not enjoy online learning as their counterparts do in other urban areas. Lack of communications access to students in rural areas is responsible for such inequality of education [23]. At the same time, while Ministry of Education has announced to study online from home, government fails to provide equal internet based education service to students in rural areas [24] particularly during Covid-19 outbreak nowadays. By adopting Technology-Community-Management model by Chib and Komathi [25], we can involve school teachers to utilize RyO Kit in providing and delivering learning materials over RyO Kit. As a result, students in rural islands will be able to study online during Covid-19 pandemic situation.

Evidently, RyO Kit is feasible to be implemented in larger scales to approach its maximum performance in filling the gap of current obstacles in delivering Covid-19 related information in rural islands of Indonesia. In addition, through its implementations in more complex environments, we would be able to identify any errors or bugs earlier which are valuable inputs for the development of next version of $\mathrm{RyO}$ Kit in the future.

\section{Summary}

This research is based on deep concern over the spread of Covid-19 in Indonesia, a country which consists of many rural islands with large populations. Lack of communication infrastructures in the area makes it difficult for government to deliver Covid-19 related information to people which in turns increase the risk of Covid-19 transmission in rural islands of Indonesia.

A novel Raspberry-Pi Outernet called RyO Kit is developed to take advantage of Outernet's potential use in tackling the issues. The RyO Kit has undergone evaluation in order to ensure its applicability by examining four parameters namely signal strength, signal quality, bit rate and quota from three different geographical locations labeled A, B, and C.

It is finally revealed in general that $\mathrm{B}$ setting point indicates better system performance than $\mathrm{A}$ and $\mathrm{C}$ ones as it outperforms them in all four parameters evaluation. In terms of signal strength, B has the highest average signal strength of $92.07 \%$. Then, for signal quality testing, B also shows best performance with average value of $69 \%$. Later for bit rate testing, B again shows top average result of $75.14 \mathrm{Kbps}$. Finally, the last testing reveals that $\mathrm{B}$ keep shows the best performance among others by having average quota of 689.64 MB.

Based on the experimental results, it is concluded that $\mathrm{RyO}$ Kit is feasible as an alternative technology to solve health information sharing issues between government and people who live in rural islands during Covid-19. Finally, recommendations offered to guide people in rural islands maximizing the benefits of the $\mathrm{RyO}$ Kit from both health and education aspects which in turn will significantly reduce potential risk of the Covid-19 spread in Indonesia.

The limitation of the RyO Kit lies in the limited quota that can be accessed which capacity is determined by the Outernet $[14,15]$. Based on our experimental results, the average quota that can be obtained by RyO Kit is $690 \mathrm{MB}$ per day. For current needs, this amount of quota is sufficient, but for greater needs in the future this limitation might be tackled by building and operating the second RyO Kit.

\section{CRediT authorship contribution statement}

Irfan Syamsuddin: Conceptualization, Methodology, Data analysis, Writing - original draft, Writing - review \& editing. Irmawati: Data collection, Data analysis, Writing review \& editing. Syafaruddin: Supervision, Reviewing.

\section{Declaration of competing interest}

The authors declare that they have no known competing financial interests or personal relationships that could have appeared to influence the work reported in this paper.

\section{Acknowledgment}

The authors would like to acknowledge support from The State Polytechnic of Ujung Pandang, Makassar, Indonesia.

\section{References}

[1] N. Wilantika, D.I. Sensuse, S.B. Wibisono, P.L. Putro, A. Damanik, Grouping of provinces in Indonesia according to Digital Divide Index, in: Proc of 6th International Conference on Information and Communication Technology, ICoICT, 2018, pp. 380-388.

[2] S. Sujarwoto, G. Tampubolon, Spatial inequality and the internet divide in Indonesia 2010-2012, Telecommun. Policy 40 (2016) 602-616.

[3] M. Roser, H. Ritchie, E. Ortiz-Ospina, Coronavirus disease (COVID19) statistics and and research, Our World in Data, 2020, https://ou rworldindata.org/coronavirus?fbclid=IwAR2qRdLFRmugoD0w_r13O4 HAOHrL1hiHfduyB2XvtXNjtQ5GGW6Dg9EsIZA. (Accessed 5 April 2020).

[4] Pandemi koronavirus di Indonesia, 2020, https://id.wikipedia.org/wiki/ Pandemi_koronavirus_di_Indonesia. (Accessed 5 April 2020).

[5] A.I. Almuttaqi, Kekacauan Respons Terhadap COVID-19 di Indonesia, The Habibie Center, 2020.

[6] S.J. Fong, N. Dey, J. Chaki, AI-empowered data analytics for coronavirus epidemic monitoring and control, in: Artificial Intelligence for Coronavirus Outbreak, in: SpringerBriefs in Applied Sciences and Technology, Springer, Singapore, 2021.

[7] D.S. Jat, C. Singh, Artificial intelligence-enabled robotic drones for COVID-19 outbreak, in: A. Joshi, N. Dey, K. Santosh (Eds.), Intelligent Systems and Methods to Combat Covid-19, in: SpringerBriefs in Applied Sciences and Technology, Springer, Singapore, 2020.

[8] A.A. Hussain, O. Bouachir, F. Al-Turjman, M. Aloqaily, AI techniques for COVID-19, IEEE Access 8 (2020) 128776-128795.

[9] J. Xie, M. Wang, R. Liu, Deep learning-based COVID-19 diagnosis and trend predictions, in: A. Joshi, N. Dey, K. Santosh (Eds.), Intelligent Systems and Methods to Combat Covid-19, in: SpringerBriefs in Applied Sciences and Technology, Springer, Singapore, 2020. 
[10] N.L. Bragazzi, H. Dai, G. Damiani, M. Behzadifar, M. Martini, J. Wu, How big data and artificial intelligence can help better manage the COVID-19 pandemic, Int. J. Environ. Res. Public Health 17 (9) (2020) 3176.

[11] A. Abiad, R. Teipelke, Infrastructure provision in developing Asia's giants: A comparative perspective on China, India, and Indonesia, J. Infrastruct. Policy Dev. 1 (1) (2017) 24-43.

[12] A.L. Pumo, Overview of the Netsukuku network, 2007, arXiv preprint arXiv:0705.0815.

[13] S. Katikala, Google Project Loon, InSight Riv. Acad. J. 10 (2) (2014) $1-6$.

[14] S. Karim, A. Rogers, E. Birrane, Bridging the information divide: Offering global access to digital content with a disruptive CubeSat constellation, in: 28th Annual AIAA/USU Conference on Small Satellites, 2014, pp. 1-8.

[15] F.S. Makhduma, C.A. Patel, S. Agrawal, OUTERNET: Crossing over data gap using cubesats, in: 2017 International Conference on Inventive Systems and Control, ICISC, 2017, pp. 1-4.

[16] M. Qu, S. Yu, D. Chen, J. Chu, B. Tian, State of the art of design, evaluation, and operation methodologies in product service systems, Comput. Ind. 77 (2016) 1-4.

[17] B. Kang, H. Choo, An experimental study of a reliable IoT gateway, ICT Express 4 (3) (2018) 130-133.
[18] M. Saari, A.M. bin Baharudin, S. Hyrynsalmi, Survey of prototyping solutions utilizing Raspberry Pi, in: 2017 40th IEEE International Convention on Information and Communication Technology, Electronics and Microelectronics, 2017, pp. 991-994.

[19] I. Syamsuddin, Setting Point RyO Kit, Patent Draft, 2020.

[20] A. Khera, Aditya, A review on the journey to Outernet, Int. J. Sci. Res. Dev. 3 (2016) 273-276.

[21] T.T.T. Edejer, Disseminating health information in developing countries: the role of the internet, BMJ 321 (2000) 797-800.

[22] Hybrid Energy for Telecom Towers, Saviva Research, 2013.

[23] K. Onitsuka, A.R.T. Hidayat, W. Huang, Challenges for the next level of digital divide in rural Indonesian communities, Electron. J. Inf. Syst. Dev. Countries 84 (2018) 12-21.

[24] Y. Bandung, A.M. Gani, H.C. Tanuwidjaja, J. Sembiring, The challenges of delivering multimedia-based learning services in rural areas, in: Proc of The 2015 International Conference on Information Technology Systems and Innovation, ICITSI, 2015, pp. 1-6.

[25] A. Chib, A.L.E. Komathi, Extending the technology-communitymanagement model to disaster recovery: Assessing vulnerability in rural Asia, in: Proc The 2009 International Conference on Information and Communication Technologies and Development, ICTD, 2019, pp. 328-336. 